\title{
OPCIONES REALES EN LA DETERMINACIÓN DE LA ÓPTIMA EDAD DE COSECHA PARA DOS MANEJOS
}

\author{
Diego Ricardo Broz ${ }^{1 *}$, Guillermo B. M. Woitschach ${ }^{1}$, Gastón Milanesi ${ }^{1}$
}

*Autor para correspondência: diego.broz@uns.edu.ar

RESUMEN: Se propone el enfoque de Opciones Reales como herramienta económica financiera para la toma de decisiones estratégicas en la determinación del turno óptimo de cosecha de dos manejos, uno de alto valor (MAV) y otro manejo pulpable (MP). Se supone que la proyección del precio de venta promedio de los subproductos sigue un proceso estocástico del tipo Geométrico Browniano, mientras que la producción se determina mediante simulación de un turno forestal. La decisión de talar la masa forestal surge de comparar en cada periodo, el valor de flujo de fondos en cada nodo $\left(F F_{i j(t)}\right)$ de una rejilla binomial con el valor esperado en el próximo año $\left(X_{t+1} \cdot e^{-\Delta t}\right)$. El enfoque de Opciones Reales, arroja que el máximo valor de ejercicio se da a los 18 años en MAV y 17 años en MP

Palabras claves: rejilla binomial, turno, opción exótica

\section{A REAL OPTIONS APPROACH TO THE DETERMINATION OF OPTIMAL HARVESTING AGE FOR TWO KINDS OF FOREST MANAGEMENT}

ABSTRACT: It was studied Real Options approach as a financial-economic tool for strategic decision-making in the determination of optimal harvesting age in two kinds of forestry exploitation, a high-value one and a pulp-producing unit. It is assumed that the projection of selling prices of the byproducts follows a Brownian geometric stochastic process, while production is determined by means of a simulation of forestry turnover. The decision of when to harvest arises from the comparison, at each period, of the cash-flow at each node $\left(F F_{i j(t)}\right)$ of a binomial lattice and its expected value in the next period $\left(\begin{array}{ll}X & e^{-\Delta}\end{array}\right)$. The Real Values approach indicates that the optimal ages are at 18 years for high-value units and 17 years for pulp producing ones.

Key words: binomial grid, shift, exotic option

\section{INTRODUCCIÓN}

Es importante contar con herramientas sólida para la toma de decisiones, entre las más importantes esta la determinación del turno óptimo de cosecha ya que es el momento en el cual el bosque se transforma de capital a renta. Bettinger et al. (2008) enumera siete tipos de rotación: física, técnica, silvicultural, biológica, por generación de ingresos, rotación económica-financiera y valoración del crecimiento porcentual.

Dentro de la rotación económico financiera encontramos variantes, estas implican actualizar el valor futuro a una tasa de riesgo sin tener en cuenta la evolución histórica de los preciso forestales, ello motiva a recurrir a una herramienta que permita incorporar dicho patrón al modelo, para ello recurrimos a las Opciones Reales ya que incluyen las variable estocástica del precio.

Los pioneros en la aplicación de componentes estocásticos en cosecha fueron Malliaris y Brock (1982), Miller y Voltaire (1983) entre otros. Thomson (1992) y Yin y Newman (1997) estudiaron el turno óptimo de cosecha asumiendo que los precios siguen un Movimiento Geométrico Browniano, este último autor menciona que los enfoques tradicionales como Faustmann se limitan a un mundo determinista.

Para Sant'Anna y Nogueira (2010) la valoración o teoría de opciones (VOR) reales es una de las herramientas mas relistas de evaluación que el valor actual neto (VAN) ya que la tasa de descuento de este permanece inalterable en la vida del proyecto al realizar el análisis. El autor recalca que en valoraciones de conseciones forestales VOR presenta valores significativamente mayores que VAN,

${ }^{1}$ Universidad Nacional del Sur - Bahía Blanca, Argentina

Cerne, Lavras, v. 20, n. 1, p. 89-96, jan./mar. 2014 
esto condice con Moreira et al. (2000), este menciona que los resultados obtenidos por VOR fueron $140 \%$ superior al calculado por VAN y mas realistas.

Matos Bentes-Gama et al. (2005) menciona que la tasa de descuento afecta significativamente el valor actual neto, siendo mas rentable el proyecto cuando menor es la tasa. La tasa de descuento no se elabora mediante un procedimiento cuantitativo riguroso, sinó mendiante un analisis cualitativo integral no riguroso o de poca consistencia condicionado por costo de oportunidad, grado de riesgo del proyecto, costo del dinero, entre otros. Esto afecta directamente al proyecto en la toma de decisión.

Se propone aplicar un enfoque de opciones reales para determinar, desde el punto de vista económico financiero, el momento más conveniente para realizar la tala en dos manejos forestales distintos. Se propone para la toma de la decisión en cuestión comparar en cada año, desde el 10 hasta el 20, el valor económico asociado al presente y el valor actual esperado de talar en el próximo año. Si el valor en el presente es mayor que el valor esperado actual, se toma la decisión de talar de lo contrario se difiere al año próximo, análogo al plateo que realiza Plantinga (1998). En términos de instrumentos financieros se considerará una opción exótica conocida como barrier option del tipo knock-in (Hull, 2005). La opción se ejerce (tala) cuando el valor del subyacente (beneficio obtenido) supera al precio de ejercicio (valor actual esperado de la tala en el próximo ejercicio). La comparación período a período es relevante porque estos valores se caracterizan por ser cambiantes en función del tiempo y riesgo de las variables.

\section{MATERIALES Y MÉTODOS}

\subsection{Método de valoración}

Las variables aleatorias relevantes son dos: la tasa de crecimiento de la masa forestal $\mathrm{E}_{(\mathrm{g})}$ y el precio de los productos obtenidos P. La evolución de la masa forestal es obtenida mediante simulación, utilizando el software Simulador Forestal (Crechi et al. 2005). Obtenido el dato del crecimiento esperado, el volumen de la masa forestal es proyectado con la (1).

$$
\operatorname{vol}_{t}=\operatorname{vol}_{t-1} \cdot\left\lfloor 1+E_{(g)}\right\rfloor
$$

Para calcular el precio promedio y la variabilidad de la mezcla de subproductos obtenidos se aplican los conceptos de la Teoría Moderna de la Cartera (Markowitz, 1991). Se emplean las ecuaciones para estimar el precio esperado (medio) y el riesgo de precio (desvío estándar) en una combinación de activos financieros. El precio medio se estima de la (2). En este caso $\mathrm{P}_{\mathrm{i}}$ representa el precio promedio de cada subproducto, $x_{\mathrm{i}}$ la participación en el total producido y $\mathrm{P}_{0}$ el precio promedio. El riesgo del precio es determinado por la (3).

$$
\begin{aligned}
& P_{o}=\sum_{i}^{n} x_{i} P_{i} \\
& \sigma_{c}=\sum_{i=1 ; j=1}^{n} x_{i}^{2} \sigma_{i}^{2}+x_{j}^{2} \sigma_{j}^{2}+2\left(x_{i} x_{j}\right) \rho_{i j} \sigma_{i} \sigma_{j}
\end{aligned}
$$

En donde $\sigma_{\mathrm{c}}$ es el riesgo del precio de la cartera de productos, $x_{i}$ y $x_{j}$, representan las participaciones de cada subproducto; $\sigma_{i}^{2}$ y $\sigma_{j}^{2}$ representan las varianzas de los precios de los subproductos; $\sigma_{i}$ y $\sigma_{j}$ los desvíos de los precios y $\rho_{i j}$ el coeficiente de correlación de precios. Usando el precio promedio de la (2) se calculan los flujos de fondos libres con la (4).

$$
F F_{t}=\left[P_{0} \cdot v l_{t}\right] \cdot\left(1-C_{t}\right) \cdot(1-I)
$$

Donde vol $_{t}$ es la producción esperada (1) y $C_{t}$ es el porcentaje que representan los costos de producción (variables y de estructura) sobre los ingresos por ventas, calculado mediante la (5). La tasa marginal de impuesto a las ganancias (I) es del 35\%. El valor intrínseco de la explotación sin flexibilidad estratégica se estima con la (6).

$$
C_{t}=\frac{c_{t}}{\left[P_{0} \cdot v o l_{t}\right]}
$$

$$
V A N_{f t}=F F_{t} \cdot e^{-k t}
$$

La (6) expresa el valor actual de los flujos de fondos derivados de la tala en cada uno de los años, descontados a la tasa ajustada por riesgo sobre inversiones equivalentes . La estructura de la tasa ad-hoc, para mercado emergente, poco diversificado y segmentado (Damodaran, 2002) surge de ajustar el rendimiento de mercado por la volatilidad total, en este caso dada por el desvío del precio. 


$$
k=(1+r m) \cdot(1+\sigma \sqrt{\Delta t})
$$

La única variable aleatoria está dada por el crecimiento de la masa forestal, suponiéndose constantes el precio promedio y los costos. La (7) muestra la formulación de la tasa ajustada por riesgo, donde $r m$ es el adicional por riesgo de mercado, $\sigma$ es la variación del precio y $\Delta t$ es la relación entre $T / n$, siendo esto la longitud de cada paso del modelo, siendo k una relación porcentual.

La proyección del precio de venta promedio de los subproductos sigue un proceso estocástico del tipo Geométrico Browniano. Se trabaja en tiempo discreto y para la elaboración de una rejilla binomial se utilizan los parámetros del modelo CRR (Cox, J- Ross, S-Rubinstein, M, 1979). Estos son:

$$
\begin{aligned}
& u=e^{\sigma c \sqrt{\Delta t}} \\
& d=e^{-\sigma c \sqrt{\Delta t}}
\end{aligned}
$$

Los movimientos de ascenso $u$ y descenso $d$ son calculados utilizando el desvío de la cartera de precios (3). El precio de cada nodo padre $(i, j)^{7}$ de la rejilla, generan los precios ascendentes $i$ y descendentes $j$ de la siguiente manera:

$$
P_{i j(t)}=\left[P_{i(t-1)} \cdot u ; P_{j(t-1)} \cdot d\right]
$$

Una vez proyectado el proceso estocástico del precio (8) y (9), se calcula nuevamente el flujo de fondos para cada periodo $(t)$ y cada nodo $(i, j)$ :

$$
F F_{i j(t)}=\left[P_{i j(t)} \cdot v o l_{t}\right] \cdot\left(1-C_{t}\right)
$$

Con los flujos de fondos para cada nodo de la rejilla se está en condiciones de construir la opción real. Para ello es menester definir el precio de ejercicio de la opción. La decisión a que se enfrenta el agente es seleccionar el momento de la tala. Para ello debe seguir un criterio de maximización de beneficios, conjugando el factor tiempo y el riesgo que emerge de las variables precio del producto y crecimiento de la masa forestal. Los flujos de fondos proyectados en cada escenario de la rejilla son comparados con el valor esperado del próximo año, según la expresión (12).

$$
X_{t+1}=\sum_{i=1 ; j=1}^{n} F F_{i j(t+1)} \cdot q_{(t+1)}
$$

El valor es el promedio de los flujos de fondos en cada nodo del próximo periodo $\left(F F_{i j(t+1)}\right)$ ponderado por la probabilidad asociada $\left(q_{i j(t+1)}\right)$. Las probabilidades asociadas a cada nodo están dadas por la (13).

$$
q_{i j(t+1)}=\frac{n !}{m !(n-m !)} \cdot\left[p^{m} \cdot\left(1-p^{n-m}\right)\right]
$$

En este caso los éxitos $(m)$ están dados por los movimientos de ascenso (subas) del precio sobre el total de observaciones $(n)$. Estas, condicionadas por los coeficientes equivalente-ciertos $(p)$, estimados con la (14).

$p=\frac{(1+r)^{\Delta t}-d}{u-d}$

Donde $r$ representa la tasa libre de riesgo y los movimientos $(u ; d)$ surgen de la (8) y (9) y $\Delta t$ tiene el mismo origen que en [8] y [9]. La decisión de talar la masa forestal surge de comparar en cada periodo, el valor correspondiente a cada nodo $\left(F F_{i j(t)}\right)$ con el valor esperado en el próximo año $\left(X_{(t+1)} \times e^{(-r \Delta t)}\right)$. Matemáticamente el problema se resume en la (15).

$$
V_{i j(t)}=\left\{F F_{i j(t)}, s i F F_{i j(t)}>X_{t+1} \cdot e^{-r \Delta t} \text { o } 0\right. \text {, sino }
$$

Si el valor de los flujos de fondos libres $\left(F F_{i j(t)}\right)$ supera al valor actual esperado del próximo año $\left(X_{(t+1)} \times e^{(-r \Delta t)}\right)$; se ejerce la tala, siendo su valor de $V_{i j}=F F_{i j(t)}$. Caso contrario; si el valor esperado para el próximo año supera los flujos de fondos libres del nodo, el resultado es de $V_{i j}=0$ difiriendo la tala al año próximo. El valor de la opción en el momento inicial $(t=0)$ requiere de utilizar el procedimiento recursivo sobre la rejilla (Milanesi 2011) dado en la (16).

$$
V_{0 t}=\sum_{i=1 ; j=1}^{n}\left\{V_{i j(t)} \cdot q_{i ; j(t)}\right\} \cdot e^{-r \Delta t}
$$

Siendo $V_{0 t}$ el valor actual de la opción de talar en el momento $(t)$. El ejercicio de la opción en cada nodo (escenario) en $(t)$ se expresa por $V_{i j(t)}$. La probabilidad de alcanzar el nodo asociado es $q_{i ; j(t)}$. El factor de actualización desde el momento $(t)$ hasta el inicio $(t=0)$ es $e^{-r \Delta t}$. Finalmente el periodo seleccionado para la tala corresponde al de mayor valor actual de beneficios futuros.

Cerne, Lavras, v. 20, n. 1, p. 89-96, jan./mar. 2014 


\subsection{Caso de aplicación}

Se propone determinar el turno óptimo de cosecha mediante la aplicación de opciones reales a dos predios forestales de 40 hectáreas cada uno de Pinus taeda L. La producción se estima mediante la simulación del crecimiento de la masa boscosa. La franja etaria de interés es a partir del año $10(t=0)$, tomando un horizonte de 10 años $(t=10)$. Los objetivos de los manejos es por un lado la producción de madera de alto valor comercial, para ello apunta a maximizar el volumen de los individuos; por otro lado se encuentra un manejo cuyo objetivo es la maximización del volumen de la masa con el objetivo prioritario de productos triturable.

En la simulación del manejo se tuvo en cuenta para el primer caso (MAV) el inicio del ciclo con 1600 plantas por hectárea, 3 intervenciones de raleos con una intensidad de $50 \%$ a $55 \%$ y periodicidad de 2 años entre intervención lo que nos permite obtener madera de alto valor favoreciendo el volumen individual (Crechi el al. 2005). La altura total de poda es de 6,5 metros. Se considera un índice de sitio de 22 metros (edad índice 15), una densidad final de 190 árboles por hectárea. Los parámetros iniciales (año 10) del bosque son: altura media 16,4 metros, diámetro medio $29,9 \mathrm{~cm}$, área basal $13,5 \mathrm{~m}^{2} / \mathrm{ha}$, un volumen de $108,7 \mathrm{~m}^{3} /$ ha y un IDR de 256 .

Para el segundo (MP) caso se tuvo en cuenta una densidad inicial de 1600 plantas/hectárea y una intervención de raleo al quinto año con una intensidad de $50 \%$. Se considera un índice de sitio de 22 metros (edad índice 15), una densidad final de 674 árboles/hectárea. Los parámetros iniciales (año 10) del bosque son: altura media 16,0 metros, diámetro medio $19,8 \mathrm{~cm}$, área basal $25,3 \mathrm{~m}^{2} / \mathrm{ha}$, un volumen de 196,4 $\mathrm{m}^{3} /$ ha y un IDR de 561 .

La simulación se lleva a cabo con el software Simulador Forestal (Crechi et al, 2005).

\section{RESULTADOS Y DISCUSIÓN}

\subsection{Estimación de los flujos de fondos}

El precio promedio inicial surge de aplicar la (2), arrojando un valor de $\$ 189,15 /$ tn para MAV y $\$ 134,07 /$ tn para MP para el periodo $t=0$. Para estimar el riesgo de precio se emplea la (3), su insumo son las series de precios históricos de los subproductos y sus respectivas participaciones. El riesgo de precio de la cartera de subproductos $\left(\sigma_{c}\right)$ es de $13,57 \%$ para MAV y $14,91 \%$ para MP. El flujo de fondos surge de aplicar la (4). Para determinar el valor del flujo es menester estimar la participación de los costos sobre ventas (5).

\subsection{Descuento de flujos de fondos}

Empleando los flujos de fondos se determina el valor actual de las potenciales talas (6). La tasa ajustada por riesgo (7) es del $28,39 \%$ para MAV y $28,73 \%$ para MP. Debido al crecimiento de la masa forestal los mayores flujos de fondos se obtienen en el último periodo. Por la sensibilidad de los beneficios al factor de actualización, el criterio del descuento de flujos de fondos indica que el mayor valor actual se produce en el instante $t=1$ para MAV y $t=0$ para MP. El resultado sugiere que la tala se realice de manera inminente. En la figura 1 se puede ver la evolución del VAN.

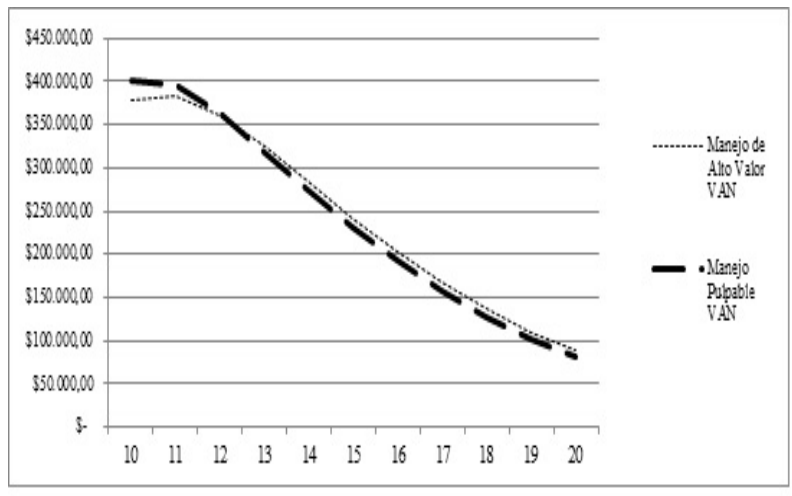

Figura 1 - Perfil de beneficios esperados con Valor Actual Neto (VAN).

Figure 1 - Profile of expected benefits according to the Net Current Value (NCV) approach.

\subsection{Opciones reales}

Para la construcción del proceso estocástico de la variable de riesgo incorporamos a los flujos de fondos el riesgo de precio. Conforme fue expresado, este asciende al $13,57 \%$ y $14,91 \%$ para MAV y MP respectivamente. El movimiento de ascenso y descenso ( 8 y 9 ) es de $u=1,1453$ y $d=0,8731$ para el MAV y $u=1,1608$ y $d=0,8615$ para el MP. Para la construcción de la rejilla se aplica la (10).

Para la construcción del flujo de fondos en la rejilla se proyectado el precio para los escenarios posibles y se estima los flujos de fondos por nodo (11).

Las probabilidades binomiales (o de ocurrencia) para cada nodo surgen de aplicar la (13). El precio de

Cerne, Lavras, v. 20, n. 1, p. 89-96, jan./mar. 2014 
ejercicio es determinado aplicando la (12). El valor del precio de ejercicio está dado por el precio promedio de los posibles resultados en cada instante de tiempo.

Para determinar el momento de la tala se recurre a los coeficientes de equivalente cierto (14), estos ascienden a $p=0,6545$ y $1-p=0,3455$ en MAV y $p=0,6341$ y $1-p$ $=0,3659$ en MP. Conforme fue expuesto, se acepta la tala en el nodo proyectado si los flujos de fondos (11) superan al valor actual esperado en el próximo ejercicio (12). Para estimar el valor actual, el precio de ejercicio es actualizado por un periodo al tipo sin riesgo, se supone una tasa $r=$ $5 \%$, para ello se utiliza la (15).

Para estimar el periodo conveniente desde el punto de vista financiero para la tala; la información de la rejilla precedente se resume en una medida de valor esperado (16). Los diferentes resultados obtenidos en cada nodo, deben ponderarse por sus probabilidades de ocurrencia (13). Con los cálculos precedentes se obtiene el valor del ejercicio (tala). Para llevarlos al mismo horizonte temporal se actualiza por el factor libre de riesgo. La decisión en juego en el último periodo se balancea entre talar o abandonar, puesto que se supone que no existe camino estratégico alternativo (venta o diferimiento).

A diferencia del criterio tradicional el enfoque de opciones reales incorpora variables estocásticas (riesgo de precio) y la flexibilidad estratégica del ejercicio de talar. El mayor valor actual se obtiene en el periodo $t=10$ (año 20). Entre los periodos $t=0$ hasta $t=9$, el máximo valor de ejercicio de la tala surge en el periodo $t=8$ (año 18) en MAV y $t=7$ (años 17) en MP. Estos resultados difieren del criterio tradicional de descuento de flujo de fondos, donde el riesgo es incorporado íntegramente en el factor de actualización, los periodos de tiempo son tratados sin flexibilidad alguna (solamente tala) y el resultado se encuentra en parte influenciado, por el impacto de la tasa ajustada por riesgo en el flujo de fondos esperado estático. En el figura 2 se presenta la evolución de los beneficios por medio de opciones reales.

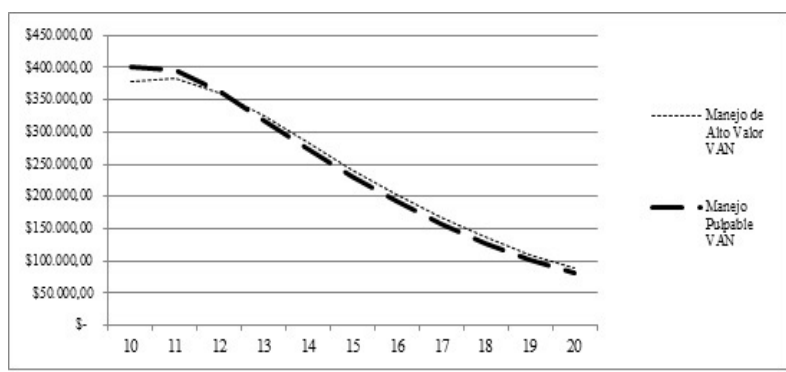

Figura 2 - Perfil de beneficios esperados con Opciones Reales.

Figure 2 - Profile of expected benefits according the Real Options Value (ROV) approach.

\subsection{Análisis de los modelos}

El método de descuento de flujos de fondos sugiere talar a los 11 años $(t=1)$ en MAV con un VAN de $\$ 382.105,6$ y a no más que 10 años $(t \leq 0)$ para MP con un VAN de $\$ 400.899,2$. Esto es una consecuencia de la alta sensibilidad negativa de los flujos al factor de descuento, lo que condice con lo mencionado por Matos Bentes-Gama et al. (2005), además de que la variable precio se supone constante en

todo el periodo de proyección. La aplicación del método de opciones reales, por el contrario, sugiere que el máximo valor se obtiene en el último periodo. Descartada esta

Tabla 1- Valores obtenidos para cada manejo donde se contrasta VAN y VOR. En gris se muestra el momento de tala.

Table 1-Values at different production types according to the NCV and ROV. Grey indicates the optimal harvesting period.

\begin{tabular}{|c|c|c|c|c|c|c|c|c|c|c|c|}
\hline & Edad masa & & 10 & & 11 & 12 & & 13 & & 14 & 15 \\
\hline \multirow{2}{*}{$\begin{array}{l}\text { Manejo de Alto } \\
\text { Valor }\end{array}$} & VAN & $\$$ & $378.224,08$ & $\$$ & $382.105,60$ & $\$ 360.674,27$ & $\$$ & $324.062,00$ & $\$$ & $283.374,08$ & \$ $241.659,44$ \\
\hline & VOR & $\$$ & - & $\$$ & - & \$ 447.278,64 & $\$$ & $371.156,38$ & $\$$ & $301.217,52$ & $\$ 722.951,00$ \\
\hline \multirow{3}{*}{ Manejo Pulpable } & VAN & $\$$ & $400.899,20$ & $\$$ & $395.929,68$ & $\$ 362.288,44$ & $\$$ & $317.971,49$ & $\$$ & $275.336,25$ & $\$ 232.058,43$ \\
\hline & VOR & $\$$ & - & $\$$ & - & $\$ 429.785,69$ & $\$$ & $340.579,83$ & $\$$ & $721.356,06$ & $\$ 640.548,88$ \\
\hline & Edad masa & & 16 & & 17 & 18 & & 19 & & 20 & \\
\hline \multirow{2}{*}{$\begin{array}{l}\text { Manejo de Alto } \\
\text { Valor }\end{array}$} & VAN & $\$$ & $202.137,47$ & $\$$ & $167.138,93$ & $136.462,11$ & $\$$ & $110.394,78$ & $\$$ & $88.657,37$ & \\
\hline & VOR & $\$$ & $643.113,21$ & $\$$ & $558.123,63$ & $\$ 983.730,13$ & $\$$ & $895.518,60$ & $\$$ & $1.909 .532,48$ & \\
\hline \multirow{2}{*}{ Manejo Pulpable } & VAN & $\$$ & $192.351,25$ & $\$$ & $157.393,72$ & \$ $127.446,04$ & $\$$ & $101.961,53$ & $\$$ & $81.063,76$ & \\
\hline & VOR & $\$$ & $551.260,82$ & $\$$ & $909.490,09$ & $\$ 824.266,90$ & $\$$ & $729.344,59$ & $\$$ & $1.651 .528,80$ & \\
\hline
\end{tabular}

Cerne, Lavras, v. 20, n. 1, p. 89-96, jan./mar. 2014 
opción, que no posee flexibilidad, el segundo momento óptimo se encuentra en el año $18(t=8)$ en MAV con un valor actual de la opción de tala de $\$ 983.730,13$ y año $17(t=7)$ con $\$ 909.490,09$ en MP, estos valores condicen con lo afirmado por Sant'Anna y Nogueira (2010) y Moreira et al. (2000). La diferencia en valor entre ambas decisiones es mas del doble, similar a lo mencionado por y Moreira et al (2000). Esto se determina en base a un proceso estocástico binomial para el precio, incorporando volatilidad en la estimación de los flujos de la rejilla para cada nodo como para el precio de ejercicio estimado. En la tabla 1 se aprecian los valores obtenidos por período con cada metodología.

\section{CONCLUSIONES}

Se expuso un modelo alternativo al clásico método de descuento de flujos de fondos, para estimar la conveniencia financiera - temporal de ejercer la opción de talar una masa forestal hipotética. Las falencia del método tradicional es no considerar la flexibilidad estratégica (diferir por un periodo) y considerar solamente el riesgo en la tasa. Se propuso aplicar la teoría de opciones reales para incorporar en el marco de decisión la posibilidad de diferir la tala y tomar en cuenta el proceso aleatorio del precio. La decisión consiste en comparar cada escenario proyectado versus el valor actual promedio de los escenarios del próximo ejercicio. En términos de opción financiera la decisión se asemeja a una barrier option del tipo knock-in.

El análisis mediante VAN genera tala prematura de las masas, 11 años en MAV y 10 años en MP no permitiendo el desarrollo futuro del bosque. En contraposición las Opciones Reales genera talas más tardías, 17 años en MAV y 18 años en MP, y con ello mayores ingresos, entre dos a casi tres veces mayores. Esto se debe a que las Opciones Reales tienen mayor flexibilidad debido a que incorpora variables estocásticas.

\section{REFERÊNCIAS}

BETTINGER, P., BOSTON, K., SIRY, J. P., GREBNER, D. L. 2008. Forest management and planning. California, 2008. 360 p.

BORISON, A. Real Option Analysis: Where are the Emperor's Clothes? Journal of Applied Corporate Finance, New York, v. 17, n. 2, p 17-31, 2005.

BRANDAO, L; DYER, J; HAHN, W. Using Binomial
Decision Trees to Solve Real Options Valuations Problems. Journal of Decision Analysis, Maryland, v. 2, n. 2, p. 69-88, 2005.

COPELAND, T; ANTIKAROV, V. Real Options. New York, 2001. 370 p.

COPELAND, T; KOLLER, T; MURRIN, J. Valuation: Measuring and Managing the Value of Companies. New York, 2000. 490 p.

COPELAND, T; WESTON, F; SHASTRI, K. Financial Theory and Corporate Policy. Boston, 2005. 1000 p.

COX, J; ROSS, S; RUBINSTEIN, M. Option Pricing: A Simplified Approach. Journal of Financial Economics. New York, v. 7, n. 3, p. 229-263, 1979.

CRECHI, E., FERNÁNDEZ, R., FRIEDL, A., \& FALHER, J. Efectos de raleo en Pinus taeda, Norte Misionero. Revista Idia, INTA, Buenos Aires, v. 11, n. 8, p 41-45, 2005.

CRECHI, E; FASSOLA, H; FRIEDL, A. Simulador Forestal. Revista Idia, INTA, Buenos Aires, v. 11, n. 8, p 46-50, 2005.

DAMODARAN, A. Investment Valuation. New York, 2002. 992 p.

HULL, J. Futures, Options and other Derivatives. New Jersey, 2005. 744 p.

MALLIARIS, A. G.; BROCKLL, W. A. Stochastic methods in economics and finance. North-Holland, Amsterdam, 1982. 303 p.

MILLER, R. A., VOLTAIRE, K. A stochastic analysis of the tree paradigm. Journal of Economic Dynamics \& Control, v. 6, n 1, p 371-386. 1983.

MARKOWITZ, H. 1991. Portfolio Selection: Efficient Diversification of Investments. Massacchusetts, 1991. $400 \mathrm{p}$.

MATOS BENTES-GAMA, M., LOPEZ DA SILVA, M., MONTOYA VILCAHUAMÁN, L. J., LOCATELLIS, M. Avalição econômica de um povoamento de

Cerne, Lavras, v. 20, n. 1, p. 89-96, jan./mar. 2014 
Eucalyptus grandis destinado a multiprodutos. Revista

Árvore, Viçosa. v. 9, n. 3, p. 401-411, 2005.

MILANESI, G. Opciones Reales: Teoria y Casos

Prácticos. Buenos Aires, 2011. 156 p.

MOREIRAA. R. B., REIS, E. J., ROCHA, K., CARVALHO, L. A valoração das concessões nas florestas nacionais da Amazônia: uma abordagem com opções reais. Pesquisa e Planeamento Econômico, Rio de Janeiro, v. 30, n. 3, p. 327-354, 2000.

PLANTINGA, A. J. The optimal timber rotation: an option value approach. Forest Science, Society of American Foresters, v. 44, n. 2, p. 192-202. 1998.

PRATT; S; GRABOWSKI; R. Cost Of Capital: Applications and Examples. New Jersey, 2008. 778 p.

PRODAN, M., PETERS, R., COX, F., REAL, P. Mensura Forestal. Costa Rica. Instituto Interamericano para la Agricultura - Gesellschaft für Zusammenarbeit (IICA-GTZ), 1997. $511 \mathrm{p}$.

SANT'ANNA, A. C., NOGUEIRA, J. M. Valoração econômica dos serviços abientais de florestas nacionais. Revista de Administração e Negócios da Amazônia, Porto Velho, UNIR, v. 2, n. 1, p. 82-108, 2010.

Sistema de Información Foresto-Industrial Provincial SIFIP: http://extension.facfor.unam.edu.ar/sifip/index. php

SMITH, J. Alternative Approach for Solving Real Options Problems. Decision Analysis, Maryland, v. 2, n. 2, p. 89-102. 2005.

THOMSON, T. A. Optimal forest rotation when stumpage prices follow a difusion process. Land Economics, New York, v 68, n 3, p 329-342. 1992.

TRIGEORGIS, L. Real Options: Managerial Flexibility and Strategy in Resource Allocations. Cambridge, 1997. 427 p.

YIN, R.; NEWMAN, D. When to cut a stand of trees? National Resour. Model, v 10, n 3, p 251-261. 1997.

Recibido: Abril 4, 2011, aceptado: 31 de julio 2013. 
